\title{
Pobreza, deforestación y sus eventuales implicaciones para la biodiversidad en Guatemala
}

\author{
LUdGer J. LOENING* \\ Michael MarkuSSEN**
}

\begin{abstract}
This paper explores the causes of deforestation and its possible implications on biodiversity loss in Guatemala. At the regional level, an empirical analysis indicates that agricultural activities remain closely tied to deforestation because of the virtual absence of non-environmental assets of the rural poor. In this context, the excessive establishment of integrated conservation and development projects appears to deliver a relatively poor outcome. The paper concludes that strengthening the non-farm sector and human capital formation should be regarded as key elements of a development strategy that tries to combine the conservation of forest biodiversity and rural poverty alleviation.
\end{abstract}

Keywords: biodiversity, deforestation, education, integrated conservation and development projects, rural poverty.

\section{Resumen}

El presente trabajo explora las causas de la deforestación y sus eventuales implicaciones para la pérdida de la biodiversidad en Guatemala. Se realiza un análisis empírico en el ámbito regional, el cual indica que las actividades agrícolas en las áreas rurales están fuertemente relacionadas a la deforestación, debido a la ausencia de activos no ambientales de los pobres, como por ejemplo la educación. En este contexto, el establecimiento excesivo de áreas protegidas dentro del país parece ser poco promedor. El trabajo concluye que el fortalecimiento del sector no agrícola y la formación de capital humano deberían tomarse como elementos claves para una estrategia de desarrollo, que intente combinar conservación de la biodiversidad forestal y reducción de la pobreza rural.

Palabras clave: biodiversidad, deforestación, educación, áreas protegidas, pobreza rural.

\footnotetext{
* Universidad de Goettingen. Correo-e: ludger.loening@wi-wiss.uni-goettingen.de

** Universidad de Goettingen. Correo-e: mmarkus@gwdg.de
} 


\section{Introducción $^{1}$}

Por medio de la ratificación del Convenio sobre la Diversidad Biológica en el año 1995, Guatemala se comprometió, entre otros temas, a llevar a cabo medidas amplias para la conservación de recursos forestales. Desde el punto de vista de un país en desarrollo, surgen varias dificultades para poner en práctica dicho convenio, ya que la disminución de la deforestación usualmente no es una prioridad social. Esta situación es problemática en la medi$\mathrm{da}$ en que es precisamente en las economías en desarrollo en las que se encuentra la mayor parte de la diversidad biológica forestal.

Como se explica en la próxima sección, el presente trabajo parte del supuesto de una estrecha relación entre el retroceso de recursos forestales y la pérdida de la biodiversidad. Una meta muy importante debe, por lo tanto, orientarse a disminuir ampliamente la deforestación, tema central del presente artículo. De acuerdo con los más recientes cálculos de la FAO (2001), la tasa anual de tala de bosques es $1.7 \%$, la cual resulta superior a la observada en Brasil o Indonesia. En el ámbito mundial, Centroamérica se cuenta como una de las regiones más afectadas por la deforestación. Schwartz (1995a, 1995b) así como Southgate y Basterrechea (1993) argumentan en este sentido, que Guatemala es un caso relevante en este tema dentro de Latinoamérica, ya que ofrece una serie de problemas característicos dentro de la región.

Con base en el planteamiento anterior, hay dos cuestionamientos principales. (a) ¿Cuáles son las razones para el drástico retroceso de recursos forestales en Guatemala? (b) ¿Qué medidas se podrían considerar como las más acertadas para la conservación del área forestal a largo plazo?

La estructura del documento es la siguiente: en el capítulo 1 se entrega una visión sobre la relación entre deforestación y la eventual pérdida de la biodiversidad en el país. Además, presentamos una visión general de la deforestación en Guatemala. El capítulo 2 habla sobre las causas directas de la deforestación y el 3 presenta los resultados empíricos sobre algunos de los factores socioeconómicos asociados. Finalmente, en el capítulo 4 se analiza cuál es la contribución de las áreas protegidas a la conservación de la biodiversidad. La conclusión se encuentra en el capítulo 5.

\footnotetext{
${ }^{1}$ Agradecemos a Adriana R. Cardozo por su valiosa asistencia, así como a los comentarios recibidos de David Kaimowitz, Norman B. Schwartz, Reginaldo Reyes Rodas, Douglas Southgate y varias discusiones con miembros del Instituto Geográfico, del Instituto de Desarrollo Rural y del Instituto Iberoamericano de la Universidad de Goettingen. Todos los errores corren bajo nuestra responsabilidad.
} 


\section{Biodiversidad y deforestación en Guatemala}

Según Myers et al. (2000) la localización natural, así como la situación biogeográfica, han llevado a que Guatemala se convierta en un centro de interés (hotspot) en el ámbito global en cuanto a la diversidad de especies. Asimismo, el Sistema de las Naciones Unidas en Guatemala (2002) coloca al país en el lugar 24 de los 25 países con mayor diversidad arbórea en el plano mundial, por lo cual algunos autores hacen énfasis en la alta prioridad de protección en el país.

Nations et al. (1989), así como Villar Anléu (1998a, 1998b), resaltan la localización del país como corredor entre las dos grandes masas continentales de tierra entre América del Norte y América del Sur, así como entre las costas Pacífica y Atlántica donde se crea una gran diversidad de flora y fauna en un espacio estrecho. De la Cruz (1982) y Holdridge (1967) mencionan que las unidades de corto espacio dependientes del relieve y la geomorfología, así como las diferencias climáticas asociadas, han permitido la construcción de una importante diversidad de ecosistemas. Particularmente, la región del Petén y los bosques de niebla de Guatemala generan una gran biodiversidad, que ha sido denominada por Dinerstein et al. (1995) como única en su clase y al mismo tiempo como muy vulnerable.

El cuadro 1 presenta una visión general sobre la deforestación en Guatemala durante los últimos 50 años. Como se puede observar, existe una fuerte tendencia a la disminución del área boscosa. Mientras en 1950 aproximadamente 65\% del área del país estaba cubierta por bosque, en el año 2000 este porcentaje descendió a $26 \%$. Igualmente, se registró una pérdida de bosque de aproximadamente $60 \%$ durante el mismo lapso. Cabe resaltar que los datos de distribución de las áreas de bosque varían considerablemente dependiendo de la fuente.

Un ejemplo son los datos para el año 1990, en los que se presentan tres estimaciones diferentes. Lo mismo sucede con las tasas anuales de deforestación. En este caso los valores varían entre 54,000 y 90,000 hectáreas por año. Al respecto no se puede descartar que los datos están influidos por el interés de cada autor al realizar los cálculos respectivos. No obstante, una valoración y revisión de los datos resulta difícil, ya que los métodos utilizados difieren considerablemente. Con base en las nuevas investigaciones de Baumeister (2001) y de la FAO (2001) respecto a la tala de árboles en el ámbito nacional, se considera como realista una ci- 


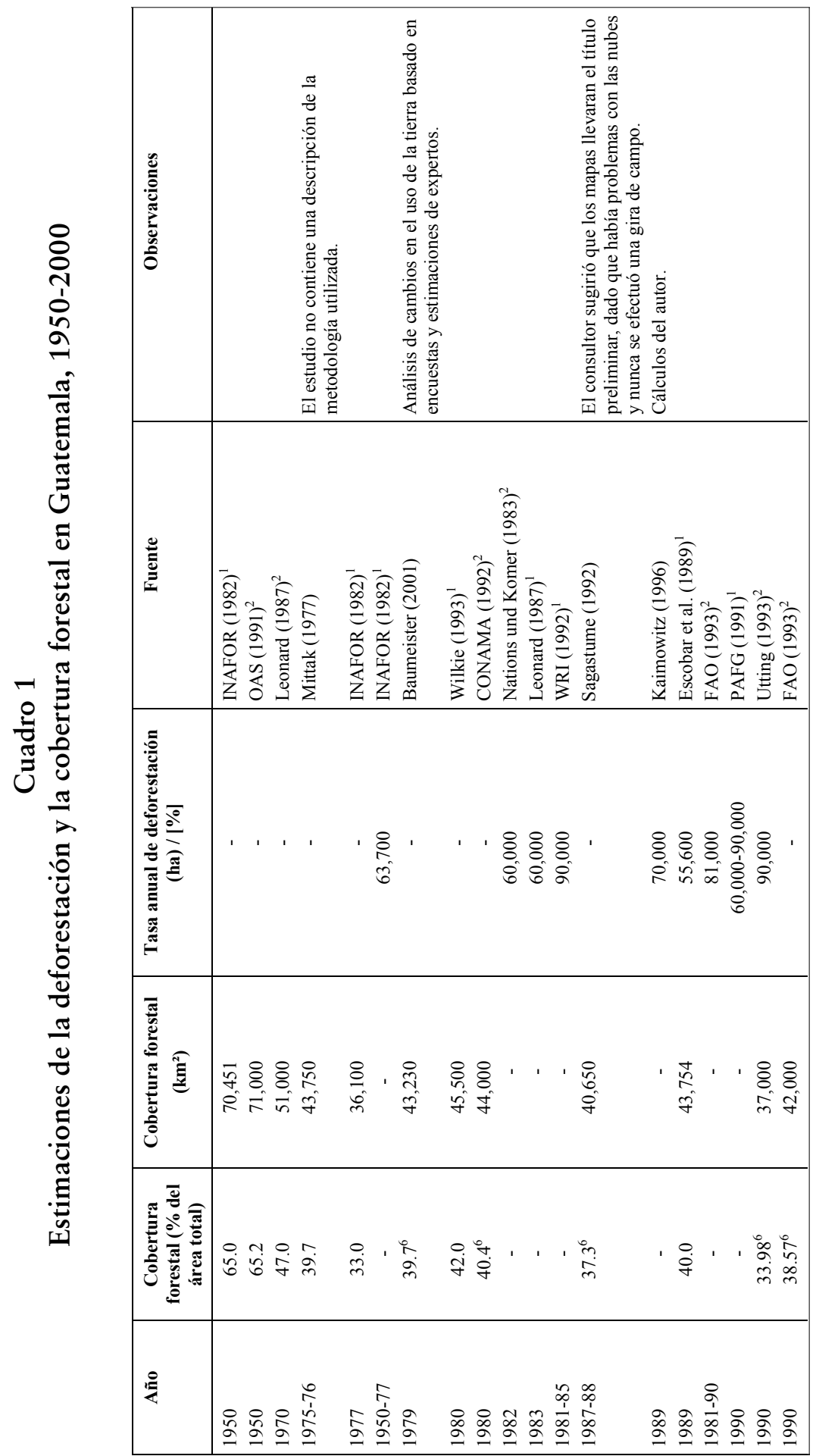




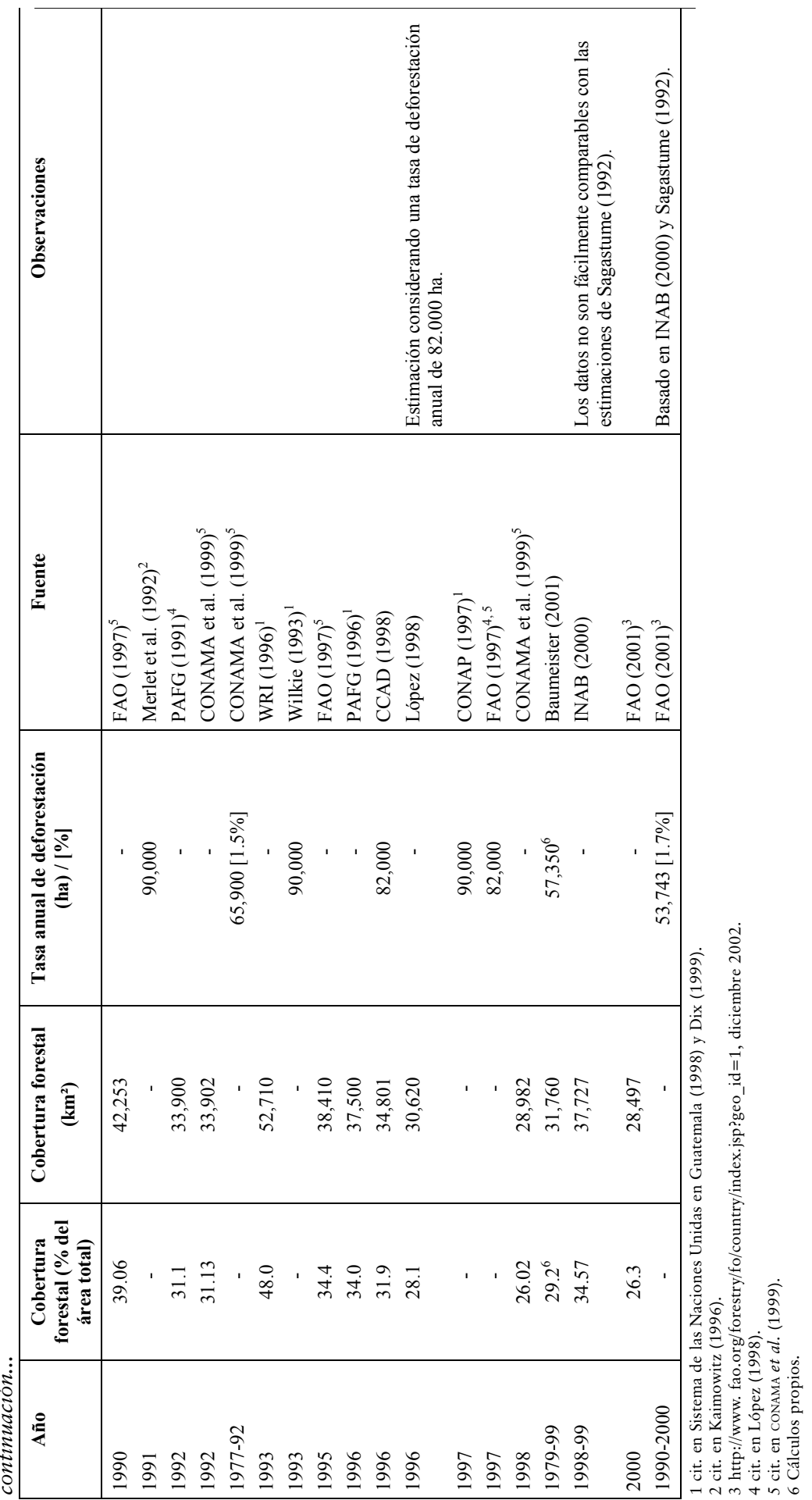


fra entre 50,000 a 60,000 hectáreas por año. Sin embargo, resulta imposible deducir de los trabajos existentes en qué dirección han sido los cambios en la tasa anual de deforestación.

La biodiversidad de especies, así como de ecosistemas en las zonas boscosas del trópico y del subtrópico, se ve amenazada por la fuerte deforestación. McArthur y Wilson (1967) presentan la clara relación entre deforestación y extinción de especies en el modelo especies-área. Lugo et al. (1993) confirman esta relación, aunque consideran que hay un riesgo de sobreestimación. Al mismo tiempo concluyen que la pérdida de la biodiversidad se puede detener por medio de estrategias administrativas, como por ejemplo la reforestación. Aunque en Guatemala algunos programas de reforestación por parte del estado se llevan a cabo, no pueden compensar el equivalente a las talas ni hacer retroceder los fuertes cambios ecológicos. Las consecuencias de la deforestación son las siguientes: pérdida directa de especies, daño y fragmentación del hábitat (Markussen y Renner 2002), consecuencias climáticas que se observan tanto en el ámbito global como en relación con el microclima, y finalmente efectos agravantes sobre las propiedades del suelo.

Usualmente en las investigaciones no se tienen en cuenta los factores abióticos, que dentro del marco del proceso de la deforestación también tienen efectos sobre la diversidad de especies. De esta forma Markussen (2003) investiga cambios pedológicos ocasionados por la deforestación en los bosques de niebla de Guatemala. Los bosques de niebla se escogieron como áreas de investigación debido a la variedad de la flora y la fauna, así como debido a los recursos naturales de agua. Las investigaciones del paisaje por medio de análisis físicos, químicos y biológicos de los suelos muestran que, a través de la deforestación de los bosques de niebla y la utilización agrícola tradicional por parte de la población Q'eqchi', se origina una degradación del suelo (véase también el anexo 2, figuras II-V). Dado que el suelo es la fuente de vida para la fauna y la flora, se generan consecuencias negativas sobre la biodiversidad forestal.

Los fuertes cambios de los parámetros químicos y físicos del suelo se pueden observar en las figuras Ia y Ib. En especial, el mantenimiento de carbono así como el nitrógeno total disminuyen fuertemente con la utilización permanente del suelo, por lo tanto, la regeneración de las áreas se ve considerablemente limitada. Para muchos organismos en el suelo, esto se traduce en la pérdida de la función de hábitat. El suelo representa la base para 


\section{Figura I}

Cambios en el nivel de nutrientes de la tierra debido a la explotación agrícola

(a) En la superficie $(0-30 \mathrm{~cm})$ de la comunidad Chelemhá, Alta Verapaz

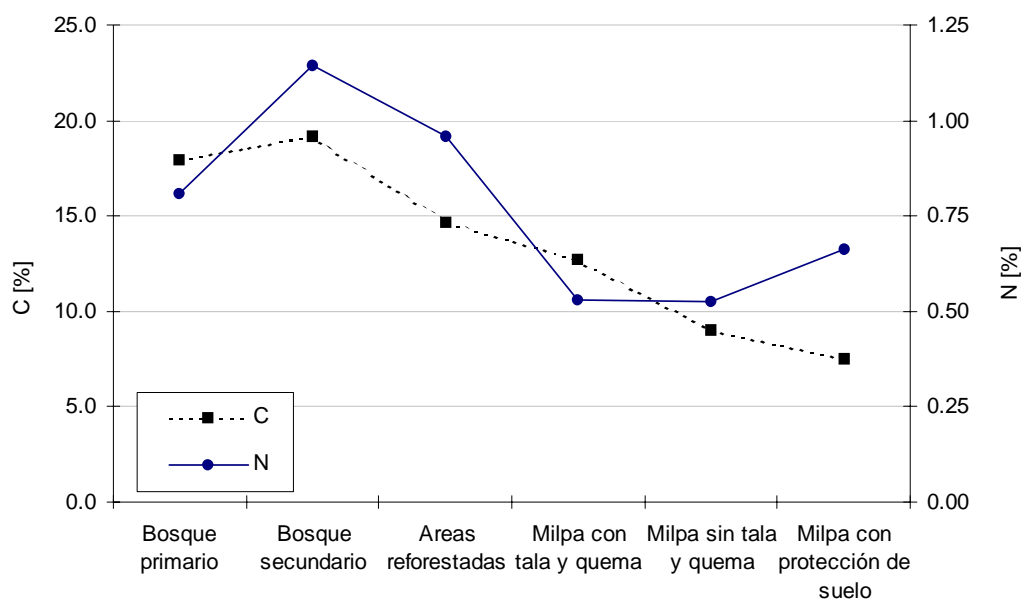

(b) En el respectivo horizonte alto (horizonte Ah) de la comunidad Chicacnab, Alta Verapaz

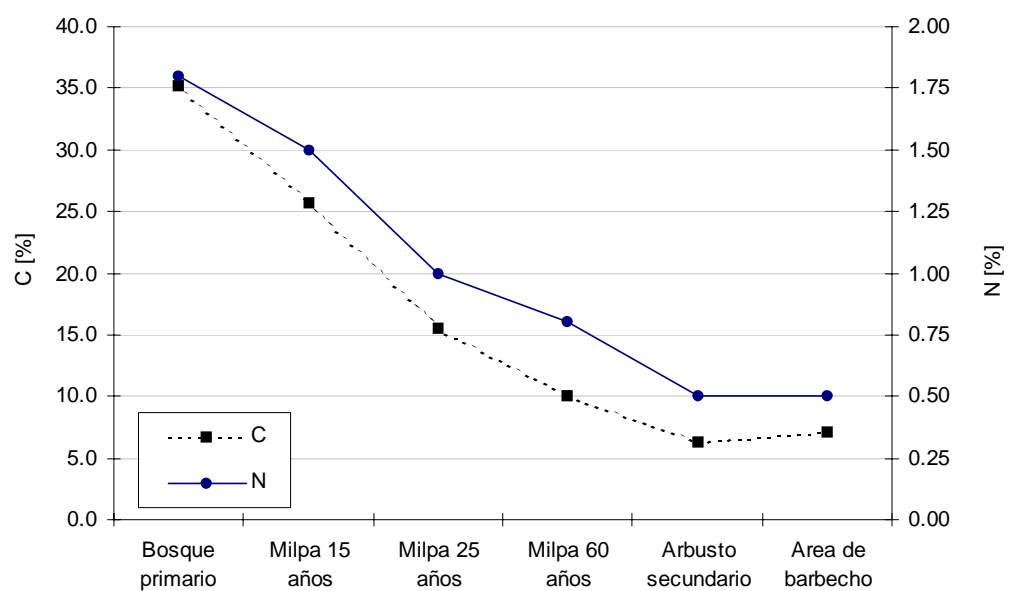

Nota: Datos propios, véase Markussen (2003) para más detalles. 
la producción primaria y toma la función de hábitat para muchos tipos de micro y meso fauna. Para la flora y la fauna que habita sobre el suelo, la degradación implica limitaciones en la función de regulación del mismo, por ejemplo limitaciones en la acumulación de energía y nutrientes. Adicionalmente, se pierde la utilidad del suelo, incluyendo la producción de alimentos para el hombre (Markussen, 2002). La degradación original del suelo iniciada por la deforestación conlleva a una caída del rendimiento de la producción agrícola, incrementando de esta forma la demanda por tierra de los pequeños agricultores y de la población que vive de la economía de subsistencia. Esta mayor demanda genera a su vez ampliación de cultivos y contribuye significativamente a la disminución de los recursos forestales. Las causas socioeconómicas de los procesos de deforestación se analizan en la siguiente sección.

\section{Causas de la deforestación}

De acuerdo con Schwartz (1995a, 1995b) se observa que históricamente la transformación de zonas forestales en zonas de producción agrícola ha jugado un papel importante en Guatemala, país considerablemente orientado a la exportación agrícola. El fuerte crecimiento económico en el sector agrícola hasta la década de los 80, intensivo en recursos naturales, contribuyó de manera significativa a la disminución del bosque. En especial, la ampliación de la economía pecuaria en el Petén fue importante para el deterioro de los recursos forestales. La consecuente devastación del bosque húmedo tropical es un claro ejemplo de decisiones políticas erradas. Según Kaimowitz $(1995,1996)$ y el Banco Mundial (1995a), las zonas consideradas improductivas fueron transformadas en tierras de pastoreo. Asimismo, se fomentó de forma activa la transformación del bosque tropical a través de adjudicaciones de grandes áreas, de créditos de bajo costo y de subvenciones.

Una recopilación de diferentes estudios sobre la deforestación en Guatemala durante los últimos 20 años se puede encontrar en la FAO (1999). Por otra parte, el Banco Mundial (1995a) y con base en el mismo Katz (2000), analiza la problemática de los derechos de propiedad de la tierra y sus efectos sobre la deforestación. En general, se observa que los estudios proporcionan resultados ambivalentes y son netamente cualitativos. Hasta los años ochenta, la mayoría de los estudios asociaba los procesos de deforestación principalmente con el aumento de la ganadería extensi- 
va. Posteriormente, el énfasis se centró en el crecimiento poblacional, la pobreza y la utilización no sostenible de la tierra como principales causantes. Cabe señalar que durante el periodo observado, sobre todo entre 1981-1983, se efectuó el conflicto interno armado que provocó fuertes movimientos migratorios. Sin embargo, según la FAO (1999) resulta difícil de evaluar el efecto neto sobre la tasa de deforestación.

Según CONAMA et al. (1999), las áreas que actualmente están sin explotar, se clasifican como inapropiadas para la producción agrícola sostenible. Dado que las áreas aptas para la producción agrícola orientada a la exportación ya se encuentran delimitadas, se sospecha que existen otras causas que han ganado significancia como generadoras de la alta deforestación. Al respecto cabe mencionar que, en el caso de Guatemala, la utilización de la madera como recurso industrial no se puede considerar como una causa importante, ya que tiene una escasa participación de $2.6 \%$ en el Producto Interno Bruto del país. Según el Sistema de las Naciones Unidas en Guatemala (2002), del total de la producción forestal entre 1995-1999, un 97\% se utilizó como combustible y sólo 3\% de la madera fue industrializada. Sorpresivamente, Guatemala (nombre que etimológicamente indica tierra de árboles) presenta una balanza comercial deficitaria en cuanto a productos forestales, cuya causa radica principalmente en la alta importación de papel y cartón.

Kaimowitz y Angelsen (2002) en un estudio acerca de la literatura disponible sobre modelos empíricos de deforestación, observan que no existe una conclusión generalizada sobre los orígenes de la deforestación. En la mayoría de los estudios se realizan análisis de corte transversal que, debido a datos dudosos, así como a la mezcla analítica de diferentes puntos de vista, ofrecen escasos avances en el conocimiento de esta problemática. Parece, por lo tanto, necesario diferenciar menores niveles de agregación entre (a) causas directas y (b) los determinantes socioeconómicos asociados.

Sin embargo, resulta irrefutable que en Latinoamérica la ampliación de las áreas de producción agrícola y sobre todo el aumento de las áreas de pastoreo han contribuido directamente a la pérdida de recursos forestales. En el caso de Guatemala, las estimaciones de expertos indican, como se observa en los cuadros 2 y 3 , una diminución de las áreas forestales debido al aumento de las necesidades alimenticias de los pequeños cultivadores. ${ }^{2}$ De acuer- 
do con estos cálculos, la tasa anual de la deforestación resulta un poco más alta que en las estimaciones de la FAO (1999).

\section{Cuadro 2}

Causas directas de la deforestación en Guatemala, 1993-1997

\begin{tabular}{|l|r|r|}
\hline \multicolumn{1}{|c|}{ Deforestación anual por } & en $\mathbf{1 , 0 0 0}$ ha & en \% \\
\hline Agricultura migratoria $^{\text {a }}$ & 64.4 & 78.5 \\
Ganadería extensiva & 8.2 & 10.0 \\
Talas ilícitas & 4.1 & 5.0 \\
Consumo de leña & 2.5 & 3.0 \\
Incendios forestales & 1.6 & 2.0 \\
Plagas y enfermedades & 0.8 & 1.0 \\
Agricultura comercial & 0.4 & 0.5 \\
\hline
\end{tabular}

Fuente: Tomado de Conama et al. (1999).

a/ La fuente no presenta una definición concreta de este término. No obstante, se asocia con actividades de tala y quema junto con migración de la población rural.

\section{Cuadro 3 \\ Guatemala: Cambios en el uso del suelo, 1979-1999}

\begin{tabular}{|l|r|r|r|}
\hline \multicolumn{1}{|c|}{ en $\mathbf{1 0 0 0}$ ha } & \multicolumn{1}{|c|}{$\mathbf{1 9 7 9}$} & \multicolumn{1}{c|}{$\mathbf{1 9 9 9}$} & \multicolumn{1}{c|}{$\Delta$} \\
\hline Superficie agrícola cosechada & 1,310 & 1,490 & 180 \\
Otras áreas agropecuarias & 3,766 & 4,638 & 872 \\
combinadas con bosque abierto & & & \\
Superficie de bosque & 4,323 & 3,176 & $-1,147$ \\
Superficie de pastos & 1,334 & 1,400 & 66 \\
Otros usos & 156 & 185 & 29 \\
\hline
\end{tabular}

Fuente: Baumeister (2001).

b/ La fuente asocia estas áreas con la expansión de la frontera agrícola y la utilización de barbechos más prolongados.

Respecto a los determinantes socioeconómicos, existen muy pocas investigaciones detalladas que analizan la relevancia empírica de la pobreza, debido principalmente a la escasa disponibilidad de datos. Sin embargo, por medio de un análisis regional para México, Deininger y Minten (1999) muestran que, junto con factores de influencia condicionados por el clima y por la calidad del suelo, las tasas de pobreza contribuyen significativamente a una mayor deforestación. Deininger y Minten (1996) comprueban 
estos resultados de forma detallada en el caso de los estados sureños mexicanos de Oaxaca y Chiapas. Debido a la presencia de características geográficas, culturales y socioeconómicas similares, estos análisis resultan altamente relevantes para Guatemala.

La pobreza y la deforestación pueden asociarse entre sí a través de la falta de ocupaciones no agrícolas. La inexistencia de otras alternativas de trabajo crea una restricción que con frecuencia obliga a ejercer actividades con ingresos bajos. Entre estas actividades se encuentra la transformación de áreas forestales en agrícolas, incluso cuando éstas no son aptas para este tipo de uso. En el caso de Guatemala, Vakis (2002) encuentra a nivel empírico que existe una fuerte relación entre actividades no agrícolas y pobreza rural. Esto se debe a tres razones. En primer lugar, existe la posibilidad de diversificar las fuentes de ingreso y por lo tanto se reduce la vulnerabilidad de los pobres hacia los choques externos. Segundo, los ingresos no agrícolas incrementan la liquidez de los agricultores, lo que les permite comprar insumos agrícolas e incrementar la productividad. Tercero, estas actividades son importantes para la población que no tiene acceso a la tierra, ya que se convierte en una alternativa de ingreso.

Pichón (1997) y Godoy et al. (1997) muestran en el contexto latinoamericano que el parámetro decisivo para las actividades de baja deforestación se encuentra en las actividades generadoras de ingreso fuera del sector agrícola. De acuerdo con Kaimowitz y Angelsen (2002), estos resultados son relevantes en la medida en que, independientemente del punto de partida analítico o empírico, en la literatura parece existir un consenso respecto a estos efectos. ${ }^{3} \mathrm{Al}$ respecto, López y Valdés (2000) destacan que en varios países latinoamericanos la educación es uno de los determinantes centrales de los ingresos agrícolas y una condición necesaria para participar en las actividades no agrícolas. Basado en la ENCOVI (Instituto Nacional de Estadística, 2000), Vakis (2002) también encuentra que para Guatemala la educación es un factor clave para participar en actividades no agrícolas en el área rural.

Cabe resaltar que, en los análisis empíricos, las tasas de pobreza basadas en el ingreso o consumo de los hogares son discutibles. Vosti y Reardon (1997) proponen por lo tanto tener en cuenta los activos de los grupos poblacionales afectados, es decir, la estructura de la propiedad de la tierra, el acceso al crédito y el capital humano, así como una variedad de factores en el contexto social e institucional. En Guatemala, dada una distribución extremadamente desigual de la tierra, infraestructura rural deficiente y el 
muy bajo nivel de capital humano, no existe para la población agrícola, mayoritariamente indígena, una alternativa diferente a realizar actividades agrícolas intensivas con baja productividad. Adicionalmente, se generan como estrategias de salida importantes movimientos migratorios hacia las regiones menos pobladas, como el Petén. Estas migraciones se originan en las altas tasas de crecimiento poblacional, que ascienden a lo largo del país a 2.6\% anual. En algunas regiones del Petén existen estimaciones que reportan tasas de crecimiento poblacional hasta de $9 \%$ anual. Surge la duda, de si estas afirmaciones se pueden comprobar empíricamente.

\section{Resultados empíricos}

Debido al estado de los datos, existen límites para la exploración empírica de los determinantes de la deforestación. Los análisis de regresión que se realizan a continuación a nivel departamental, con excepción de Ciudad de Guatemala, deben por lo tanto leerse bajo esta salvedad. La regresión en la cual se basa el análisis es:

$$
\Delta \mathrm{DEF}_{\mathrm{i}}=\mathrm{C}+\beta_{\mathrm{ij}} \cdot \mathrm{X}_{\mathrm{ij}}+\mathrm{DUMMY}_{\text {Petén }}+\mathrm{u}_{\mathrm{i}}
$$

Donde $\triangle \mathrm{DEF}$ es deforestación per capita, c es una constante, $\beta_{\mathrm{ij}}$ es el vector de coeficientes y $\mathrm{X}_{\mathrm{ij}}$ representa el vector de la variable explicativa $\mathrm{j}$ en la región $\mathrm{i}$. Por su parte $\mathrm{u}_{\mathrm{i}}$ es el término de error. Las variables explicativas incluyen el área total de las microfincas, la pobreza extrema rural, el rendimiento de maíz como una variable proxy para la productividad agrícola, el empleo rural no agrícola, la educación y una variable dummy para capturar los efectos específicos de la región del Petén (véase el cuadro 4). Una breve descripción de las variables y sus fuentes se puede encontrar en el anexo 1 (cuadros 8 y 9). Dada la limitada disponibilidad de datos, el modelo econométrico resulta relativamente simple y parte del supuesto de una relación lineal entre las variables. Sin embargo, los resultados generales han probado ser robustos. La utilización de especificaciones logarítmicas o semilogarítmicas no cambiaría significativamente los resultados. ${ }^{4}$ Ya que hay evidencia de heterocedasticidad, el test $t$ se corrige utilizando el procedimiento de White.

La regresión indica una relación significativa entre el consumo per capita de recursos forestales y entre la pobreza rural (columna 1). El signo positivo del coeficiente respecto a la pobreza seña- 
la que con una proporción creciente de la población rural en condiciones de extrema pobreza, se da un incremento de la deforestación per capita a nivel departamental. Las cuotas regionales de pobreza y las definiciones correspondientes fueron tomadas de una investigación realizada por el Banco Mundial (1995b). La extrema pobreza indica que aproximadamente $72 \%$ de la población rural no está en capacidad de cubrir las necesidades mínimas diarias de calorías. En una revisión posterior, el Banco Mundial (2003) ha reducido esta estimación en aproximadamente 10 puntos porcentuales.

Debido a que los problemas de distribución de la tierra en Guatemala juegan un papel importante, en la columna (1) y en las regresiones siguientes, se incorpora el área total de microfincas, es decir, de unidades de producción agrícola hasta 0.7 hectáreas. Aunque la variable indica una relación significativa, no hay una explicación concreta para este resultado. Por lo tanto, se podría aducir el signo negativo a la diferente distribución de los derechos de propiedad en el ámbito regional. En este sentido, las regiones en las que los derechos de propiedad están mejor regulados, registran una menor deforestación. No obstante, se observa una gran deficiencia en la regulación de derechos de propiedad a lo largo del país. Posiblemente la variable indica también que, junto con la explotación de pequeñas parcelas, se llevan a cabo paralelamente otras labores. Para la población predominantemente indígena resulta de gran importancia tener la posibilidad de cultivar el maíz, planta tradicional, lo que se sale de una interpretación netamente económica. En muchas regiones del país, las áreas de producción de este tamaño son insuficientes para la alimentación de una familia.

Adicionalmente a las variables mencionadas, se colocó en la columna (2) la tasa de retorno del maíz. En presencia de muchos factores influyentes, por ejemplo la calidad del suelo y las condiciones climáticas, no resulta sorpresiva la baja significancia. Es por que las escasas y variables relaciones climáticas conllevan a diferentes tasas de retorno entre las regiones. Mientras que en algunas regiones es posible llevar a cabo una sola cosecha por año, en las regiones más abundantes se pueden hacer varias. No obstante, el signo de la regresión tiende a indicar la importancia de la aplicación de nuevas y mejores técnicas de producción agrícola, con el fin de alcanzar incrementos en la productividad. El signo negativo implica que, al haber un mayor retorno del maíz, existe una menor deforestación per capita a lo largo del país. Sin 


\section{Cuadro 4 \\ Determinantes de la deforestación en Guatemala, 1975-1988}

\begin{tabular}{|c|c|c|c|}
\hline \multirow[t]{2}{*}{ Regresión por MCO } & \multicolumn{3}{|c|}{$\begin{array}{l}\text { Variable dependiente: } \\
\text { Deforestación per capita }\left(\mathrm{km}^{2}\right)\end{array}$} \\
\hline & (1) & (2) & (3) \\
\hline Constante & $\begin{array}{l}-0.687 \\
(-0.35)\end{array}$ & $\begin{array}{l}0.699 \\
(0.32)\end{array}$ & $\begin{array}{l}4.191 \\
(1.54)\end{array}$ \\
\hline Area de microfincas $\left(\mathrm{km}^{2}\right)$ & $\begin{array}{c}-0.058 * * \\
(-3.84)\end{array}$ & $\begin{array}{l}-0.043 * * \\
(-2.75)\end{array}$ & $\begin{array}{c}-0.058 * * \\
(-3.38)\end{array}$ \\
\hline $\begin{array}{l}\text { Población rural en pobreza extrema } \\
\text { (participación) }\end{array}$ & $\begin{array}{l}6.429 * \\
(1.94)\end{array}$ & $\begin{array}{l}5.049 \\
(1.52)\end{array}$ & \\
\hline $\begin{array}{l}\text { Empleo rural no agrícola } \\
\text { (participación) }\end{array}$ & & & $\begin{array}{l}-4.586 * * \\
(-2.64)\end{array}$ \\
\hline Escolaridad promedio (años) & & & $\begin{array}{l}-1.639 * \\
(-2.22)\end{array}$ \\
\hline Rendimiento de maíz (qq/mz) & & $\begin{array}{l}-5.500 \\
(-1.27)\end{array}$ & \\
\hline Dummy Petén & $\begin{array}{c}14.472 * * \\
(19.96)\end{array}$ & $\begin{array}{l}15.070 * * \\
(21.03)\end{array}$ & $\begin{array}{l}13.818 * * \\
(22.15)\end{array}$ \\
\hline $\mathrm{R}^{2}$ ajustado & 0.814 & 0.814 & 0.864 \\
\hline Estadístico F & 30.11 & 22.91 & 32.83 \\
\hline $\mathrm{N}$ & 21 & 21 & 21 \\
\hline \multicolumn{4}{|c|}{$\begin{array}{l}\text { Entre paréntesis se encuentran los valores t corregidos por heterocedasticidad } \\
\text { usando el método de White. } \\
* \text { significativo al } 5 \% \text {. } \\
* * \text { significativo al } 1 \% \text {. }\end{array}$} \\
\hline
\end{tabular}

Fuente: Cálculos propios.

embargo, en la frontera agrícola no es indispensable la incorporación de estas técnicas, ya que su efecto puede ser variado, e incluso podrían provocar un aumento de la deforestación (véase la sección 4), dependiendo de diversos factores que no se analizan en detalle en este documento. 
En la columna (3) se reemplaza finalmente la variable pobreza por la participación de la ocupación rural en el sector no agrícola, así como por la educación promedio. El signo negativo indica que tanto una mayor educación promedio, como una mayor participación de la ocupación rural en el sector no agrícola, generan una menor deforestación per cápita. Ambas variables afectan levemente el $\mathrm{R}^{2}$ y concuerdan con el valor total de la variable de pobreza. La ausencia de posibilidades de ocupación no agrícolas, junto con el bajo capital humano rural, pueden verse como causas centrales de la pérdida de recursos forestales en Guatemala dentro del modelo empírico. Así mismo, se confirma un resultado cualitativo para Guatemala obtenido en la investigación de C. Cabrera (1996). De igual manera, Godoy y Contreras (2001) concluyen en una investigación sobre indígenas a nivel de hogares en Bolivia, que el parámetro más importante para la menor deforestación es el nivel educativo. Posibles causantes de la relación anterior pueden ser, junto con mejores oportunidades de empleo, la mayor valoración de los recursos forestales o mejoras en la productividad. A través de un mayor nivel de capital humano, podrían generarse, además, movimientos migratorios en áreas urbanas, lo que disminuiría la presión sobre la utilización de la tierra.

La región del Petén resulta muy relevante debido a su tamaño. Por esta razón se incorporan, a través de una variable dummy, efectos regionales específicos en el análisis. Se deben diferenciar dos efectos: (a) mientras en años anteriores los programas de fomento estatal, y a través de éstos la ampliación de la tierra de pastoreo, jugaron un papel importante, (b) los estudios de Bilsborrow (1992), así como los de Sader et al. (2001) indican, por medio de fotos satelitales, que actualmente los procesos de colonización de los pequeños agricultores son el principal causante de la deforestación. Estos resultados se confirman en Grunberg et al. (2000) mediante la aplicación de modelos empíricos de pronóstico para conversión de bosques. Resulta interesante que el ingreso agrícola no juegue ningún papel significativo en el incremento en la capacidad de pronóstico del modelo. Las razones para la migración rural en el Petén pueden, por lo tanto, ser indirectamente asociadas con la conversión de los bosques. 


\section{Cuadro 5}

Motivos para la migración rural en Guatemala, 1995-2000 a/

\begin{tabular}{|l|r|r|}
\hline \multicolumn{1}{|c|}{ en \% } & \multicolumn{1}{c|}{ Total } & \multicolumn{2}{|c|}{ El Petén } \\
\hline Bajos ingresos & 4.6 & 4.5 \\
Falta de empleo en el sector agrícola & 18.8 & 63.6 \\
Falta de empleo en el sector no agrícola & 10.3 & 4.5 \\
Razones familiares & 34.5 & 20.5 \\
Matrimonio & 13.8 & 4.5 \\
Otras razones & 18.0 & 2.3 \\
\hline
\end{tabular}

a/ Cambio de domicilio de la población agrícola de más de veinte años en una finca propia o con actividades agrícolas.

Fuente: Cálculos propios basados en la ENCOvi (2000).

\section{Cuadro 6 \\ Razones para trasladarse al Petén, 1999}

\begin{tabular}{|l|r|r|}
\hline \multicolumn{1}{|c|}{ en \% } & A Petén & Dentro de Petén \\
\hline Buscar tierra & 65.4 & 48.4 \\
Vivir con familia o amigos & 17.8 & 15.8 \\
Buscar un trabajo no agrícola & 4.7 & 6.1 \\
Tener acceso a educación, servicios de & n.a. & 5.1 \\
salud o agua & & \\
Escapar de inseguridad o violencia en otro & 1.4 & 2.4 \\
lugar & & 22.4 \\
Otras razones & 9.3 & \\
\hline
\end{tabular}

Fuente: INE et al. (2001).

En el cuadro 5 se enumeran algunos motivos para la migración de la población rural con actividades agrícolas en fincas cultivadas de manera autónoma. La migración rural se refiere al cambio de domicilio dentro de los límites de un municipio. Para el total del país, estas variables son poco explicativas dentro del contexto de la deforestación. No obstante, dado que el Petén tiene altas tasas de inmigración de otros municipios, es importante analizar estos resultados. Aproximadamente dos tercios de los encuestados en el Petén colocan la falta de empleo en el sector agrícola como la principal causa de la migración. El poco significado de ingreso respecto a formas alternativas de empleo no resulta sorprenden- 
te, ya que se asocia con el bajo nivel educativo. Estos resultados son confirmados en el cuadro 6. Como se puede observar, la gran mayoría de los inmigrantes vinieron a Petén para buscar tierra por alguna u otra razón.

En este sentido, se podría realizar una contribución importante a la conservación de la cobertura forestal y a la disminución de la pobreza a través de la construcción de capital humano y del fomento de alternativas de ingreso en actividades no agrícolas, como se explica en secciones anteriores. Con base en la dimensión temporal y en un determinado escepticismo frente a la eficacia de las políticas de desarrollo, se favorecen con frecuencia propuestas alternativas para la protección directa de la biodiversidad forestal. La contribución de las áreas protegidas, tanto públicas como privadas, se discute en la siguiente sección.

\section{4. ¿Cómo contribuyen las áreas protegidas a la conservación de la biodiversidad?}

Generalmente se argumenta que a través de las áreas protegidas se puede hacer una contribución significativa a la conservación de los bosques y de la biodiversidad en los países en desarrollo. A través del apoyo de donantes extranjeros, Guatemala le ha dado un papel importante a la creación de parques naturales. Según el Sistema de las Naciones Unidas en Guatemala (2002), existen más de 123 áreas protegidas en el país. La mayoría de éstas fueron creadas a principios de los noventa y corresponden aproximadamente al 28.6\% del territorio nacional.

Las áreas protegidas son administradas normalmente por el Consejo Nacional de Areas Protegidas (CONAP), entidad pública dependiente de la Presidencia de la República de Guatemala. En algunos casos organizaciones no gubernamentales (ONG) u otras instituciones son aceptadas como coadministradoras, por medio de un convenio firmado entre la organización y el CONAP. Uno de los proyectos más significativos, que contempla aproximadamente un tercio del área en mención, es la Reserva de la Biósfera Maya en el Petén. Según C. Cabrera (1996) la problemática agraria del país se ha agudizado gracias a la masiva ampliación de los parques naturales y del retorno de desplazados por la guerra civil. Por esta razón, no es de sorprender que existan tensiones entre los promotores de las áreas protegidas y la población rural. 
Gran parte de las áreas protegidas en Guatemala se ejecutan en forma de planes integrales de protección de la naturaleza. De esta manera se intenta evitar conflictos sociales, a través del establecimiento de una zona de amortiguamiento. Igualmente surgen, bajo el razonamiento de integración entre protección y utilización, fuentes adicionales de ingreso para la población local, que incentivan a la población a abandonar las prácticas nocivas de utilización de la tierra. Ejemplos al respecto son el ecoturismo y la adopción de técnicas de agricultura sostenible. No obstante, Valenzuela (1996) afirma que, en el caso de Guatemala, la mentalidad de protección de la naturaleza se encuentra generalmente en primer plano.

Si se tiene en cuenta la alta popularidad de las áreas protegidas, resulta sorprendente que muchos estudios como el realizado por Wells et al. (1992), indiquen que los proyectos de protección integrada de la naturaleza no han alcanzado en la mayoría de los casos las metas planteadas inicialmente en materia de conservación del medio ambiente y desarrollo. Las investigaciones de Barret y Arcese (1995), Gibson y Marks (1995), Alpert (1996), Southgate (1998), Oates (1999), Wells et al. (1999) y Rice et al. (2001), muestran que en los países en desarrollo las debilidades institucionales, en conjunto con problemas conceptuales no resueltos, son los responsables del amplio fracaso de la mayoría de las áreas protegidas.

Entre los principales obstáculos conceptuales se encuentran tres factores. En primer lugar, las medidas de desarrollo incentivan la inmigración en las zonas de amortiguamiento, lo que incrementa la presión sobre la utilización de la tierra. En segundo lugar, las mejoras en la productividad agrícola liberan mano de obra a través de la adopción de técnicas de agricultura sostenible, lo que genera efectos negativos sobre la conversión de bosques. En general, evaluar los efectos de aumentos en la productividad agrícola sobre la deforestación es aún un tema controvertido (Angelsen y Kaimowitz 2001). Finalmente, en el caso del subempleo se cuestiona si a través de la participación en proyectos, por ejemplo de ecoturismo, se reducen realmente las prácticas intensivas de utilización de la tierra.

En este sentido, Brandon (1996) resalta que el ecoturismo en los países en desarrollo tiene sólo una importancia marginal. El éxito de este concepto requiere de una serie de criterios que con frecuencia no se cumplen. Especialmente la exclusividad de la belleza natural y la disponibilidad de una infraestructura turística 
juegan un rol importante. Según Southgate (1998), el supuesto éxito del modelo costarricense es un buen ejemplo de que el ecoturismo no incrementa significativamente las fuentes de ingreso de la población local.

Por el contrario, en el caso de México, Deininger y Minten (1999) concluyen que las áreas protegidas han contribuido significativamente a la disminución de la deforestación. No obstante, el efecto cuantitativo en comparación con los factores socioeconómicos y geográficos es muy bajo. Asimismo, Bruner et al. (2001) concluyen, en un estudio comparativo, que las áreas protegidas contribuyen a la reducción de la tasa de deforestación. Por su parte, Southgate (1998) critica que, en vista de la constelación de intereses por parte de las instituciones dirigentes, existe una tendencia a una presentación enaltecedora de las áreas protegidas, lo que dificulta la discusión y evaluación real de las mismas. A esto se suma que la mayor parte de la información proviene de consultores con intereses propios y que usualmente los datos socioeconómicos y cartográficos necesarios para realizar una evaluación no se encuentran disponibles. Para resumir, la discusión sobre la valoración del éxito o fracaso de las áreas protegidas aún está abierta.

En el caso de Guatemala, Godoy (1998) así como Friedrich (2002) señalan importantes deficiencias institucionales y administrativas en las zonas protegidas. Adicionalmente, muchos parques naturales existen solamente en el papel. Según el Sistema de las Naciones Unidas (2002), en 1997 solamente 13\% del total de áreas protegidas declaradas contaba con un plan maestro aprobado y en ejecución. Este porcentaje subió significativamente en los últimos años, y actualmente se estima que $87 \%$ de las áreas protegidas cuenta con un plan maestro. Sin embargo, aún ante la existencia de una administración más eficiente, es poco probable que las áreas protegidas alcancen sus objetivos, debido a las causas mismas de la deforestación, así como a las deficiencias conceptuales mencionadas anteriormente.

Ya que las áreas protegidas son numerosas y heterogéneas, es difícil realizar una ilustración detallada de todas. No obstante, la Reserva de la Biósfera Maya del Petén constituye un caso de particular interés, ya que ha sido objeto de diversas investigaciones en los últimos años. Una excelente recopilación se puede encontrar en Nations et al. (1999) y FLACSO (2000). Basados en fotos satelitales, Sader et al. (2001) encuentran que la deforestación anual de la Reserva de la Biosfera Maya ha aumentado continua- 
mente en los últimos años (véase el cuadro 7). Asimismo, se registran incrementos en casi todas las zonas protegidas, así como en las zonas de amortiguamiento. ${ }^{5}$

\section{Cuadro 7 \\ Deforestación anual en la Reserva de la Biosfera Maya, 1986-1997}

\begin{tabular}{|l|c|c|c|c|}
\hline \multicolumn{1}{|c|}{ en \% } & $\mathbf{1 9 8 6 - 1 9 9 0}$ & $\mathbf{1 9 9 0 - 1 9 9 3}$ & $\mathbf{1 9 9 3 - 1 9 9 5}$ & $\mathbf{1 9 9 5 - 1 9 9 7}$ \\
\hline Total & 0.04 & 0.23 & 0.33 & 0.36 \\
Zonas de & 0.74 & 2.71 & 3.76 & 3.28 \\
amortiguamiento & & & & \\
\hline
\end{tabular}

Fuente: Sader et al. (2001).

Como explicación, Chemonics International et al. (2000) sostienen que la utilización de técnicas alternativas de producción agrícola no conduce a menores actividades de tala. Además, las zonas de amortiguamiento crean un corredor de servicios para las áreas protegidas, con lo que incrementa la conversión de bosques. De manera similar, los Defensores de la Naturaleza (2001) afirman que en la Reserva de la Biósfera Sierra de las Minas, la adopción de técnicas de agricultura sostenible tiende a aumentar la deforestación, lo que difiere del pensamiento convencional. Asimismo, se confirma la inquietud expresada por Valenzuela (1996), según la cual los proyectos de protección que se plantean con buenos propósitos, muchas veces tienen resultados ambivalentes. En este sentido, Shriar (2001) argumenta que se debería hacer más énfasis en los aspectos del desarrollo de la economía rural en el Petén.

Llama la atención que a partir de 1990, es decir, a partir del establecimiento de la reserva, hay un importante incremento en la deforestación. Sin embargo, queda en duda si la deforestación hubiese sido mayor en ausencia de las áreas de protección y las zonas de amortiguamiento. Si se tiene en cuenta el cambio en la utilización de la tierra en el Petén, en regiones no cobijadas por la protección, se constata que la deforestación resulta mayor que en las áreas protegidas y en las zonas de amortiguamiento, resultado que también se observa en el Parque Nacional Laguna Lachuá en Alta Verapaz. En la foto satelital se puede observar la frontera del parque nacional con una magnitud de 14.500 ha. Mientras que el parque aún se encuentra casi por completo cubierto por bosque, las zonas aledañas fueron deforestadas casi por completo durante los últimos años. De esta manera, resulta positiva la protección 
del bosque dentro del parque nacional, aunque falta manejo de las zonas de amortiguamiento.

Por su parte, las reservas naturales privadas brindan ventajas en cuanto a la protección de la biodiversidad forestal. Schulz y Unger (2000) destacan como un buen ejemplo al respecto el Proyecto Ecológico Quetzal, que se desarrolla en el Departamento Alta Verapaz. Según Friedrich (2002), las fortalezas se encuentran especialmente en la red de áreas protegidas, el cambio en las formas de explotación, el ecoturismo y la utilización sostenible de los bosques, cada uno a nivel de pequeñas unidades familiares agrícolas. La protección privada de la naturaleza, llevada a cabo por ONG o entidades privadas, es un instrumento útil para cumplir con el Convenio sobre la Diversidad Biológica. Una ventaja importante radica en la posibilidad de controlar los límites del área, lo cual se garantiza especialmente cuando la ong está domiciliada en el lugar y a través de su presencia puede actuar con mayor facilidad en la región del proyecto. Cabe resaltar que para el establecimiento y conservación de las áreas protegidas, la participación de la población adyacente juega un papel importante. De igual manera, resulta importante la sensibilización de la población infantil escolar respecto a la protección de la naturaleza, a través de la cual se garantizan multiplicadores para el resto de la población en el futuro. También E. F. Cabrera (1996) concluye que una de las prioridades para la conservación de la biodiversidad forestal en Guatemala es la educación ambiental, la cual debería fomentarse en gran escala junto con la ampliación de la concientización respecto al valor de la biodiversidad.

Otro ejemplo en cuanto a protección de la naturaleza se encuentra en la ONG Unión para Proteger el Bosque Nuboso (UPROBON). A través de la compra de un área de bosque de niebla intacto y con el objeto de reforestar en el marco del Programa de Incentivos Forestales (PINFOR) en los puntos degradados, se garantiza la protección de los recursos forestales y la consecuente biodiversidad. La meta principal en este programa es la adquisición y tramitación de posibilidades para crear una base de vida con fuentes alternativas de ingreso para la población indígena, sin que sea necesario recurrir a la deforestación. La población indígena aprende las principales funciones del bosque de niebla y se sensibiliza respecto a la utilización sostenible de los recursos. Hasta ahora, las reservas naturales privadas constituyen menos del 1\% de la superficie de las áreas protegidas (Sistema de las Naciones Unidas en Guatemala 2002). Sin embargo, la protección privada de la 
naturaleza que es aún escasa en Guatemala, podría ofrecer un cierto potencial.

\section{Conclusiones y necesidades futuras de investigación}

(1) Según el Convenio sobre la Diversidad Biológica, Guatemala se comprometió a fomentar la protección de ecosistemas y áreas naturales, así como a proteger las poblaciones en su entorno natural. Adicionalmente, se debe promover el desarrollo sustentable que contemple el medio ambiente en zonas adyacentes a las áreas protegidas. Aproximadamente una tercera parte de Guatemala se encuentra bajo protección, sin embargo, se pueden reconocer deficiencias graves en la puesta en marcha del convenio.

(2) Las reservas naturales privadas podrían ser aptas para la conservación de la biodiversidad forestal. Una ventaja es que ante la existencia de problemas específicos, podrían plantearse soluciones locales. Dentro de este contexto muchos actores consideran que la puesta en marcha de sistemas sostenibles de utilización de la tierra es favorable, en especial si se adaptan a las condiciones locales. En ambos casos, la promoción de capital humano, y en especial la inversión en educación ambiental, constituyen una posibilidad de afrontar la pérdida de la biodiversidad forestal.

(3) A nivel de las áreas protegidas manejadas por instituciones gubernamentales u ONG, resulta poco prometedora una política de conservación de bosques que no contemple los incentivos que afectan la utilización intensiva de la tierra, en especial aquellos condicionados por reglas institucionales o por factores relacionado con la pobreza. En vez de concentrarse solamente en el establecimiento de áreas protegidas, con dudosa probabilidad de éxito, es necesario mejorar las capacidades administrativas y buscar alternativas para la conservación de la cobertura forestal.

(4) Dado que en Guatemala los factores asociados con la pobreza juegan un papel importante, es indispensable fortalecer los activos de los grupos socialmente marginados, en especial los indígenas. Para mejorar los activos hay varias posibilidades; sin embargo, medidas como la construcción de infraestructura física, la facilitación de acceso a los sistemas de crédito, las reformas agrarias y de propiedad, así como la adopción de técnicas de agricultura sostenible son discutibles, ya que pueden tener resultados ambivalentes, y provocar incluso un aumento en la deforestación. El conocimiento preciso de estas relaciones, así como su significa- 
do empírico, debería por lo tanto ser de interés para investigaciones futuras.

(5) Guatemala ofrece condiciones óptimas para profundizar la investigación empírica. Existen mapas digitales para los años 1988 (Sagastume 1992) y 1999 del Instituto Nacional de Bosques (INAB, 2000), con los cuales se pueden calcular tasas de deforestación como las realizadas por la FAO (2001). Además, la Universidad del Valle de Guatemala está elaborando actualmente, en conjunto con otras instituciones, una documentación sobre la dinámica de la cobertura forestal entre 1991 y 2001. Adicionalmente, existe una completa base cartográfica nacional digitalizada, elaborada por el Ministerio de Agricultura, Ganadería y Alimentación (MAGA, 2001). Estas informaciones pueden ampliarse a través de las encuestas sociodemográficas del Instituto Nacional de Estadística para los años 1989, 1998 y 2000.

(6) Un elemento central de una política de desarrollo orientada a la pobreza radica en mejorar la educación de la población rural, ya que a través de esto se amplia la posibilidad de entrar en el mercado laboral del sector agrícola no rural. Al mejorar las opciones de ingreso, disminuye el incentivo para realizar labores intensivas en recursos, así como en utilización de la tierra, que se asocian generalmente con una baja productividad. Al contrario de las posibilidades ya mencionadas para mejorar los activos de los pobres, se encontró a nivel empírico que existe una relación inequívoca e importante para Guatemala respecto al fomento de capital humano. Además, la educación juega un papel importante en la disminución del crecimiento poblacional, así como posiblemente en el mejor conocimiento y en la apreciación de los recursos naturales.

(7) Bajo las condiciones actuales, en Guatemala no parece existir un conflicto de metas entre la conservación de la biodiversidad forestal y el crecimiento económico. Por el contrario, el crecimiento económico surge como una condición necesaria para la transformación del sector agrícola, así como para disponer de otras alternativas de empleo. El fomento de capital humano es una posibilidad para cambiar el curso del crecimiento económico del país, basado en el uso de recursos naturales y que, según el Banco Mundial (2003), hasta hoy no ha sido muy exitoso en la reducción de la pobreza. Asimismo, Loening (2002) muestra empíricamente que la formación de capital humano es indispensable para aumentar a largo plazo la tasa de crecimiento económico. 
Anexo 1

Datos

\section{Cuadro 8 \\ Fuentes de datos para el modelo de regresión}

\begin{tabular}{|c|c|c|}
\hline Variable & Fuente & Anotación \\
\hline $\begin{array}{l}\text { Cobertura forestal en } \\
1975 / 6\end{array}$ & Mittak (1977) & $\begin{array}{l}\text { El estudio no contiene una } \\
\text { descripción de la metodología } \\
\text { utilizada. }\end{array}$ \\
\hline $\begin{array}{l}\text { Cobertura forestal en } \\
1987 / 8\end{array}$ & Sagastume (1992) & $\begin{array}{l}\text { El consultor sugirió que los mapas } \\
\text { llevan el título preliminar dado que } \\
\text { había problemas con nubes y que } \\
\text { nunca se efectuó una gira de campo. }\end{array}$ \\
\hline $\begin{array}{l}\text { Cobertura forestal en } \\
1998 / 9\end{array}$ & INAB (2000) & $\begin{array}{l}\text { Bien que la FAO (2001) utilizó los } \\
\text { mapas de INAB (2000) y de } \\
\text { Sagastume (1992) para elaborar la } \\
\text { tasa de deforestación al nivel } \\
\text { nacional, los datos al nivel regional } \\
\text { no son fácilmente comparables, } \\
\text { impidiendo así un análisis } \\
\text { estadístico. }\end{array}$ \\
\hline Población & $\begin{array}{l}\text { Dirección General de } \\
\text { Estadística (1981) }\end{array}$ & $\begin{array}{l}\text { El Censo de } 1981 \text { se efectuó durante } \\
\text { el conflicto armado interno. Sin } \\
\text { embargo, los resultados han sido } \\
\text { considerados robustos utilizando } \\
\text { datos de otros años. }\end{array}$ \\
\hline Área de microfincas & SEGEPLAN (1984) & $\begin{array}{l}\text { Basado en el Censo Agropecuario de } \\
1979 .\end{array}$ \\
\hline $\begin{array}{l}\text { Población rural en pobreza } \\
\text { extrema }\end{array}$ & Banco Mundial (1995b) & $\begin{array}{l}\text { Basado en la Encuesta Nacional } \\
\text { Socio-Demográfica de 1989. Posible } \\
\text { sobreestimación de las cifras. }\end{array}$ \\
\hline $\begin{array}{l}\text { Población rural ( } \geq 15 \text { años) } \\
\text { en actividades no agrícolas }\end{array}$ & $\begin{array}{l}\text { Instituto Nacional de } \\
\text { Estadística (1994) }\end{array}$ & $\begin{array}{l}\text { Ha sido considerada la mejor fuente } \\
\text { de información a pesar del año. }\end{array}$ \\
\hline Rendimiento maíz & SEGEPLAN y PNUD (1993) & $\begin{array}{l}\text { Sobre la base de la Encuesta } \\
\text { Agrícola de Granos 1986/7. }\end{array}$ \\
\hline Promedio de escolaridad & Banco Mundial (1996) & $\begin{array}{l}\text { Basado en la Encuesta Nacional } \\
\text { Socio-Demográfica de } 1989 .\end{array}$ \\
\hline
\end{tabular}




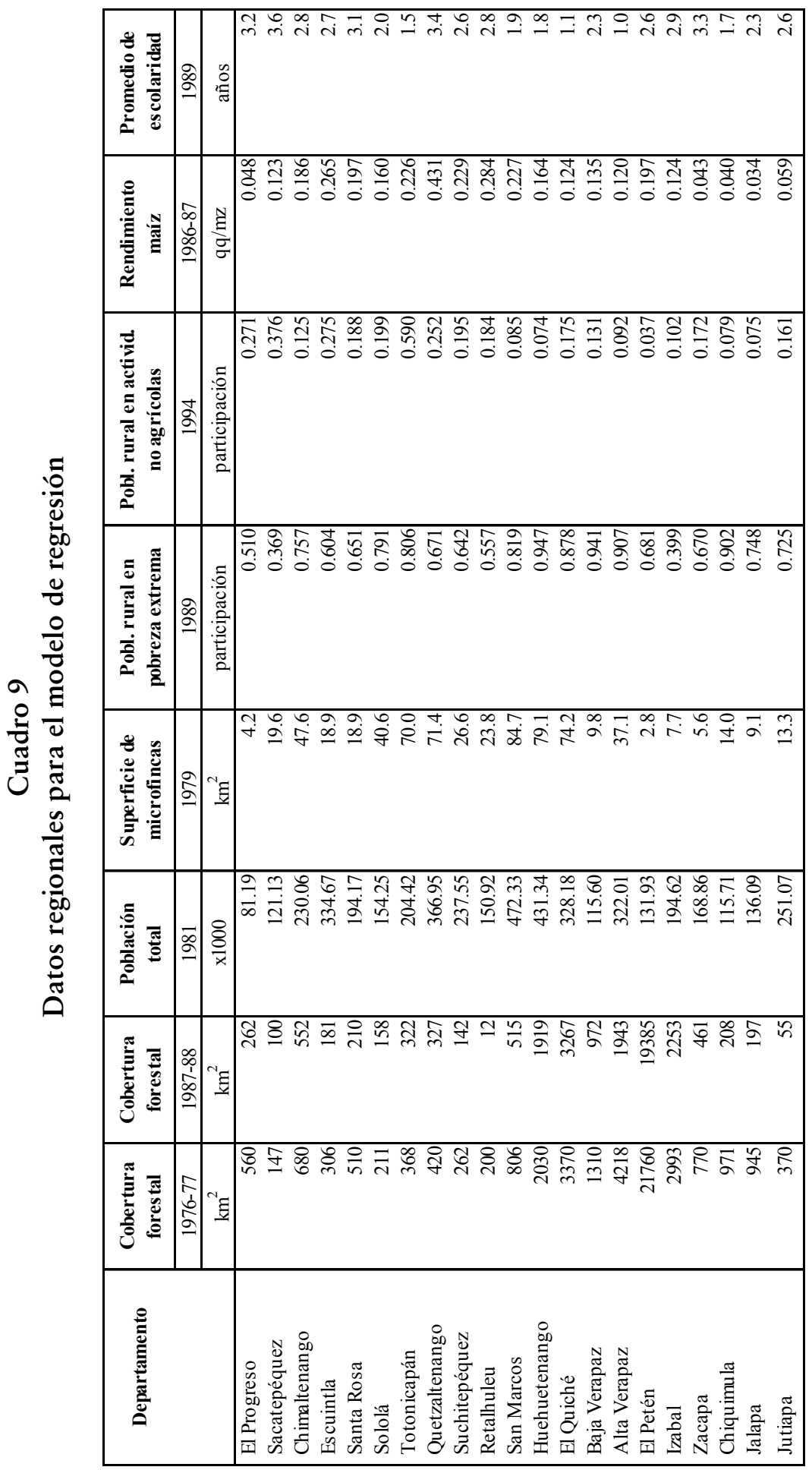




\section{Anexo 2}

\section{Foтоs}

\section{Figura I}

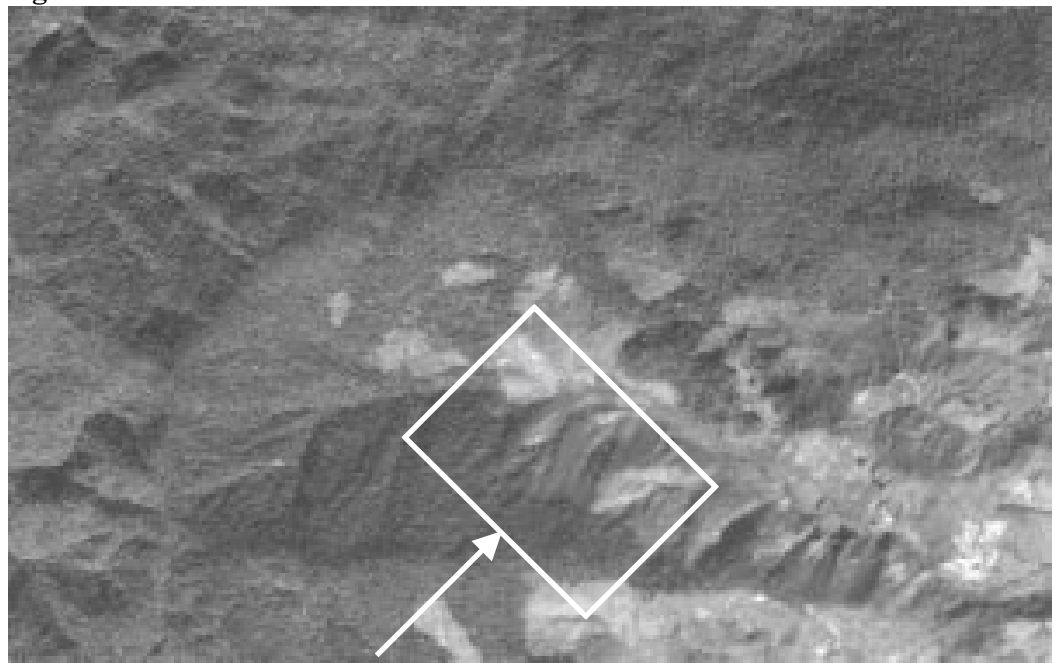

Comunidad Chelemhá (municipio Tucurú, departamento Alta Verapaz), 1964. La colonización ya está en plena actividad y la fragmentación del hábitat apenas empieza (Fuente: Instituto Geográfico Nacional Guatemala, VV Hurd M-6 AMS1-23-64 AID2 1022).

\section{Figura II}

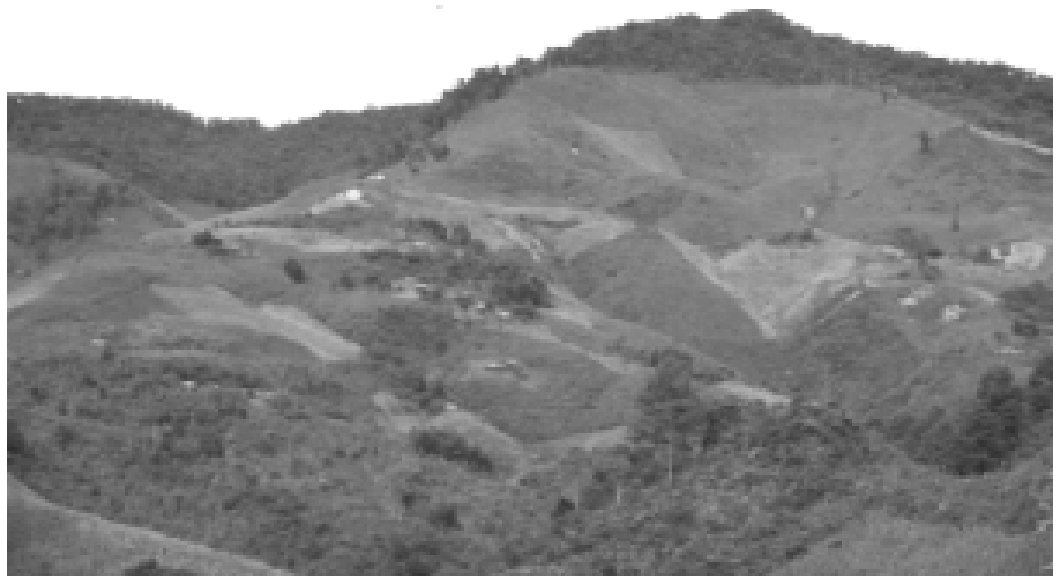

La misma comunidad Chelemhá con parcelas de milpa y formas de erosión del año 1998. Se observan bosques solamente en la parte superior de las colinas. El borde del bosque está a la altura de $1900 \mathrm{~m}$. 


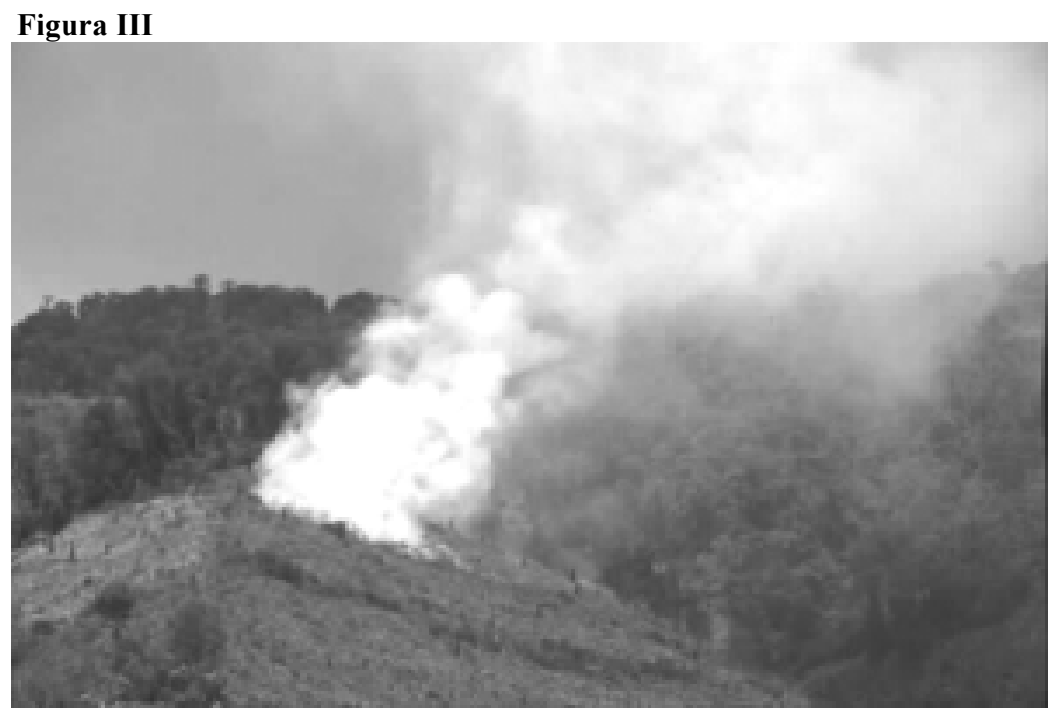

Al fin de la estación seca, los campesinos usualmente queman las parcelas para el cultivo nuevo (Chelemhá en 2001).

\section{Figura IV}

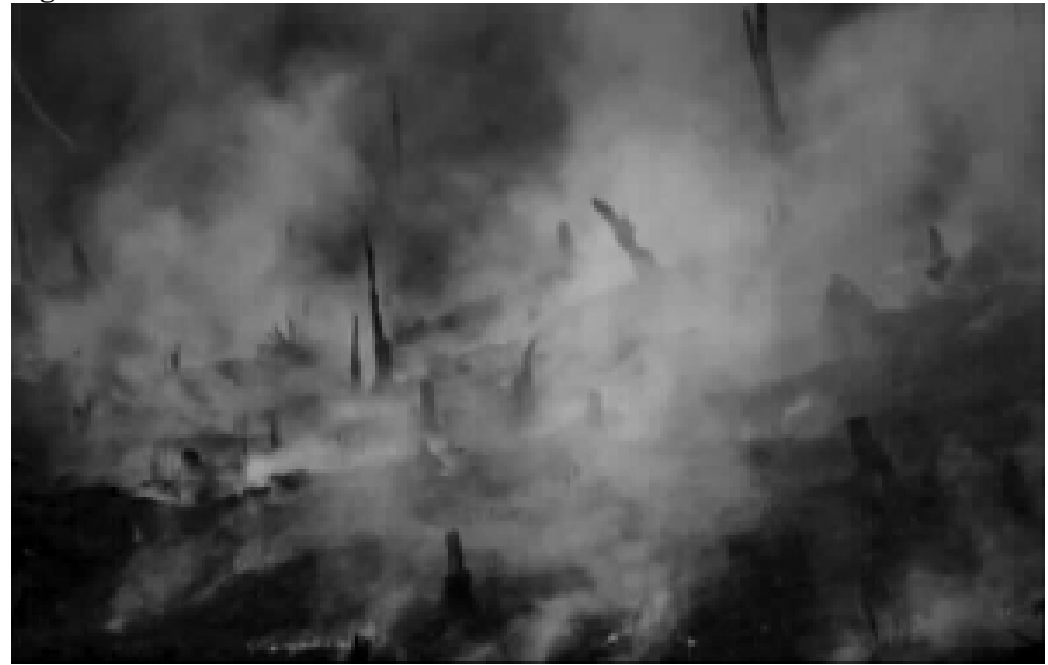

Desmonte tradicional por incendio para preparar la milpa (Chelemhá en 2001). Fuente de las figuras I-IV: autores. 


\section{Bibliografía}

Alpert, P. (1996), "Integrated Conservation and Development Projects”, en BioScience, año 46, núm. 11, pp. 845-855.

Angelsen, A. y D. Kaimowitz (eds.) (2001), Agricultural Technologies and Tropical Deforestation, Bogor, Center for International Forestry Research.

Banco Mundial (2003), Poverty in Guatemala, reporte núm. 24221-GU, Washington D.C.

- (1996), Guatemala Basic Education Strategy: Equity and Efficiency in Education, reporte núm. 13304-GU, Washington D.C.

- (1995a), Guatemala: Tenencia Agraria y Manejo de los Recursos Naturales, reporte núm. 14553-GU, Washington D.C., mimeo.

- (1995b), Guatemala: An Assessment of Poverty, reporte núm. 12313-GU, Washington D.C.

Barret, C. y P. Arcese (1995), "Are integrated conservation-development projects sustainable? On the conservation of large mammals in sub-Saharan Africa", en World Development, núm. 23, pp. 1073-1084.

Baumeister, E. (2001), "Formas de acceso a la tierra y al agua en Guatemala”, en Cuadernos del Desarrollo Humano, núm. 4, Sistema de las Naciones Unidas, Guatemala.

Bilsborrow, R. (1992), "Rural Poverty, Migration, and the Environment in Developing Countries: Three Case Studies", en World Bank Policy Research Paper 1017.

Brandon, K. (1996), "Ecotourism and Conservation: A Review of Key Issues”, en World Bank Environment Department Paper, núm. 33. 
Bruner et al. (2001), "Effectiveness of Parks in Protecting Tropical Biodiversity”, en Science, núm. 291, pp. 125-128.

Cabrera, C. (1996), Síntesis histórica de la deforestación en Guatemala, Facultad Latinoamericana de Ciencias Sociales, Guatemala, mimeo.

Cabrera, E. F. (1996), Diagnostico sobre la gestión de los recursos de biodiversidad y la aplicación de la Agenda 21 en Guatemala: Proyecto Manejo Sostenible de Recursos Naturales, mimeo.

CCAD (Comisión Centroamericana de Ambiente y Desarrollo) (1998), Estado del ambiente y los recursos naturales en Centroamérica 1998, San José, Costa Rica.

Dirección General de Estadística (1981), Censos Nacionales. IV Habitación y IX Población, Características Generales, Guatemala, (publicado en 1984).

Instituto Nacional de Estadística (1994), X Censo Nacional de Población y IX de Habitación, Características Generales de Población y Habitación, Guatemala, (publicado 1996).

Chemonics International BIFOR Consortium y International Resources Group (2000), Guatemala: Assessment and Analysis of Progress Toward s05 Goals in the Maya Biosphere Reserve, Guatemala, reporte final de consultoría a USAID, 6 de diciembre del 2000.

CONAMA et al. (Comisión Nacional de Medio Ambiente) (1999), Las áreas silvestres de Guatemala, ¿Tienen amenazas? Guatemala, mimeo.

CONAP (Consejo Nacional de Áreas Protegidas) (1997), El estado de la Reserva de la Biósfera Maya en 1996, Guatemala, CONAP/AID/Fondo Peregrino, mimeo.

De la Cruz, J. R. (1982), Clasificación de zonas de vida de Guatemala a nivel de reconocimiento, Ministerio de Agricultu- 
ra, Ganadería y Alimentación e Instituto Nacional Forestal, Guatemala, mimeo.

Defensores de la Naturaleza (2001), Impacto de la Agricultura Sostenible sobre la Conservación de la Biodiversidad: Reserva de la Biósfera Sierra de las Minas, Guatemala, Biodiversity Support Program, Washington, D.C.

Deininger, K. W. y B. Minten (1999), "Poverty, policies, and deforestation: The case of Mexico" en Economic Development and Cultural Change, año 47, núm. 2, pp. 313-344.

(1996), Determinants of Forest Cover and the Economics of Protection: An Application to Mexico, World Bank Research Project on Social and Environmental Consequences of Growth-Oriented Policies, Working Paper núm. 10.

Dinerstein, E. et al. (1995), A Conservation Assessment of the Terrestrial Ecoregions of Latin America and the Caribbean, Banco Mundial, Washington, D.C.

Dix, A. (1999), "Balance de los recursos naturales", en TorresRivas, E. y J. A. Fuentes (eds.): Guatemala: las particularidades del desarrollo humano. Volumen II: Sociedad, medio ambiente y economía, Sistema de las Naciones Unidas, Guatemala, pp. 311-356.

Instituto Nacional de Estadística (2000), Encuesta Nacional Sobre Condiciones de Vida (Encovi), Guatemala (publicado en 2002).

Escobar, J. y C. Rodríguez (1989), Situación de los recursos forestales de Guatemala, El primer congreso Forestal Nacional, Colegio de Ingenieros Agrónomos, Guatemala, mimeo.

FAO (2001), State of the World's Forests, Roma.

- (1999) Annotated Bibliography Forest Cover Change Guatemala, Forest Resources Assessment Programme, Working Paper núm. 13, Roma. 
- (1993), Forest Resources Assessment 1990, Tropical Countries, faO Forestry Paper núm. 112, Roma.

FLACSO (Facultad Latinoamericana de Ciencias Sociales) (2000), Nuevas Perspectivas de Desarrollo Sostenible en Petén, Guatemala.

Friedrich, S. (2002), "Schutz der biologischen Vielfalt durch Schutzgebiete in Guatemala? Akzeptanzprobleme und Lösungsansätze an drei Beispielen”, en Bundesamt für Naturschutz (ed.), Treffpunkt Biologische Vielfalt II, Bonn, pp. 245-252.

Gibson, C. y S. Marks (1995), “Transforming rural hunters into conservationists: an assessment of community-based wildlife management programs in Africa”, en World Development, núm. 23, pp. 941-957.

Godoy, J. (1998), “Matrices de Proyectos en Ejecución y un Perfil Conceptual”, en Diagnóstico Resumido del sIgAP, Guatemala, informe para USAID, capítulo 4, pp. 25-27, mimeo.

Godoy, R. et al. (1997), "Household Determinants of Deforestation by Amerindians in Honduras", en World Development, núm. 25, pp. 977-987.

Godoy, R. y M. Contreras (2001), “A Comparative Study of Education and Tropical Deforestation among Lowland Bolivian Amerindians: Forest Values, Environmental Externality, and School Subsidies", en Economic Development and Cultural Change, año 49, núm. 3, pp. 555-574.

Grunberg, W., D. Guertin y W. Shaw (2000), Modeling Deforestation Risk for the Maya Biosphere Reserve, Guatemala, documento presentado en Twentieth Annual ESRI International User Conference, San Diego, California, mimeo.

Holdridge, C. (1967), Life Zone Ecology, San José, Costa Rica, Tropical Science Center Publications. 
INAB (Instituto Nacional de Bosques) (2000), Informe Final, Fase I: Mapa de Cobertura Forestal para 1999, Guatemala, mimeo.

INAFOR (Instituto Nacional Forestal) (1982), Memoria de labores 1982, Guatemala, mimeo.

INE (Instituto Nacional de Estadística) et al. (2001), Guatemala, Salud, Migración y Recursos Naturales en Petén, Resultados del Módulo Ambiental en la Encuesta de Salud Materno Infantil 1999, Guatemala.

Kaimowitz D. y A. Angelsen (2002), Economic Models of Tropical Deforestation: A Review, Bogor, Center for International Forestry Research, tercer borrador, mimeo.

Kaimowitz, D. (1996), Livestock and Deforestation in Central America in the 1980s and 1990s: A Policy Perspective, Bogor, Center for International Policy Research, mimeo. Kaimowitz, D. (1995), Land Tenure, Land Markets, and Natural Resource Management in the Petén and Northern Transversal of Guatemala. Bogor, Center for International Policy Research, mimeo.

Katz, E. (2000), "Social Capital and Natural Capital: A Comparative Analysis of Land Tenure and Natural Resource Management in Guatemala", en Land Economics, año 76, núm. 1, pp. 114-132.

Leonard, H. (1987), Natural resources and economic development in Guatemala, New Brunswick, International Institute for Environment and Development.

Loening, L. J. (2002), The Impact of Education on Economic Growth in Guatemala: A Time-Series Analysis Applying an Error-Correction Methodology, Discussion Paper núm. 87, Ibero-America Institute for Economic Research, University of Goettingen.

López, P. (1998), Potencial de carbono y absorción de dióxido de carbono de la biomasa en pie por encima del suelo en los 
bosques de la República de Guatemala, informe de consultoría, Guatemala, mimeo.

López, R. y A. Valdés (eds.) (2000), Rural poverty in Latin America, New York, St. Martins Press.

Lugo, A., J. A. Parrotta y S. Brown (1993), "Loss in Species Caused by Tropical Deforestation and Their Recovery Through Management”, en Ambio, año 22, núm. 2-3, pp. 106-109.

MAGa (Ministerio de Agricultura, Ganadería y Alimentación) (2001), Mapas digitales a escala 1:250.000 elaborados por el Sistema de Información Geográfica (SIG-MAGA), CD-ROM, Guatemala.

Markussen, M. (2002), Pedoökologische Folgen des mennonitischen Landnutzungssystems im Oriente Boliviens (Departamento Santa Cruz), Stuttgart, ibidem-Verlag.

Markussen, M. (2003), “GIS-gestützte Bewertung des Landnutzungspotenzials zur Schutzgebietsausweisung in Bergnebelwäldern Guatemalas”, en Bundesamt für Naturschutz (eds.): Treffpunkt Biologische Vielfalt III, Bonn.

y S. C. Renner (2002), "Landschaftsveränderungen und avifaunistische Diversität in einem guatemaltekischen Bergnebelwald in Alta Verapaz", en Verhandlungen der Gesellschaft für Ökologie, núm. 32, Changing Landscapes, Changing Ecology, p. 302.

McArthur, R. H. y E. O. Wilson (1967), The Theory of Island Biogeography, Monographs in Population Biology núm. 1, Princeton University Press, Princeton.

Merlet, M. et al. (1992), Identificación de un programa regional de desarrollo sostenible en el trópico húmedo, informe de consultoría, Groupe de Recherche et d'Echanges Technologiques, Paris, mimeo.

Mittak, W. L. (1977), Guatemala. Estudios para la reforestación nacional. Fortalecimiento al sector forestal, documento de trabajo núm. 25, PNUD/FAO, Guatemala, mimeo. 
Myers, N. et al. (2000), "Biodiversity hotspots for conservation priorities”, en Nature, núm. 403, pp. 853-858.

Nations, J. D. et al. (1999), Thirteen Ways Of Looking at a Tropical Forest, Washington D.C., Conservation International.

Nations, J. D., B. Houseal, I. Ponciano et al. (1989), Biodiversidad en Guatemala: Evaluación de la diversidad biológica y los bosques tropicales, Washington D.C.

Nations, J. D. y D. Komer (1983), "Rainforests and the Hamburger Society”, en Environment, año 25, núm. 3, pp. 12-20.

Oates, J. (1999), Myth and Reality in the Rain Forest: How Conservation Strategies Are Failing in West Africa, Berkeley, University of California Press.

Organization of American States (OAS) (1991), República de Guatemala: Proyecto de Manejo y Conservación de los Recursos Naturales Renovables de la Cuenca del Río Chixoy, Washington D.C.

PAFG (Plan de Acción Forestal) (1991), Plan de Acción Forestal de Guatemala, Documento base y perfiles de proyectos, Guatemala, PAFG/AID, mimeo.

Pichón, F. (1997), “Colonist Land-Allocation Decisions, Land Use, and Deforestation in the Ecuadorian Amazon Frontier", en Economic Development and Cultural Change, año 44, núm. 4, pp. 707-744.

Rice, R. et al. (2001), Sustainable Forest Management: A Review of Conventional Wisdom, Washington D.C., Center for Applied Biodiversity Studies.

Sader, S. A. et al. (2001), "Forest monitoring of a remote biosphere reserve", en International Journal of Remote Sensing, año 22, núm. 10, pp. 1937-1950.

Sagastume, R. (1992), Informe Mapa de la Cubierta Forestal de la República de Guatemala, Guatemala, Plan de Acción Forestal, mimeo. 
Schulz, U. y D. Unger (2000), “Integration von Landnutzung und Regenwaldschutz - eine Fallstudie aus Guatemala”, en Landnutzungsplanung und Naturschutz, Berlin, Wissenschaft und Technik-Verlag, pp. 94-105.

Schwartz, N. B. (1995a), “Colonization, Development and Deforestation in Petén, Northern Guatemala", en Painter, M. y W. H. Durham (eds.): The Social Causes of Environmental Destruction in Latin America, University of Michigan Press, Ann Arbor, pp. 101-130.

- (1995b), Forest Society: A Social History of Petén, Guatemala, $3^{a}$ edn., University of Pennsylvania Press, Philadelphia.

SEgEPlan (Secretaría General del Consejo Nacional de Planificación Económica) (1984), Recopilación de Información Comparativa de los Censos Agropecuarios 1950, 1964 y 1979 y Series de Superficie, Producción y Disponibilidad por Rubros Agrícolas, 1950-1982, Guatemala, mimeo.

- y PNUD (Programa de la Naciones Unidas para el Desarrollo) (1993), Dimensión, Entorno y Consecuencias de la Pobreza, Guatemala, mimeo.

Shriar, A. J. (2001), “The Dynamics of Agricultural Intensification and Resource Conservation in the Buffer Zone of the Maya Biosphere Reserve, Petén, Guatemala”, en Human Ecology, año 29, núm. 1, pp. 27-48.

Sistema de las Naciones Unidades en Guatemala (1998), Informe Nacional de Desarrollo Humano 1998, Guatemala: Los Contrastes del Desarrollo Humano, Guatemala.

Sistema de las Naciones Unidas en Guatemala (2002), Guatemala: Desarrollo Humano, Mujeres y Salud, Informe Nacional de Desarrollo Humano 2002, Guatemala.

Southgate, D. (1998), Tropical Forest Conservation. An Economic Assessment of the Alternatives in Latin America, New York, Oxford University Press. 
Southgate, D. y M. Basterrechea (1993), "Population Growth, Public Policy and Resource Degradation: The Case of Guatemala”, en Ambio, año 21, núm. 7, pp. 460-464.

Utting, P. (1993), Trees, People and Power. Social Dimensions of Deforestation and Forest Protection in Central America, Geneva, United Nations Research Institute for Social Development y World Wildlife Fund.

Vakis, R. (2002), Guatemala: Livelihoods, Labor Markets, and Rural Poverty, Guatemala Poverty Assessment Program, World Bank Technical Paper núm. 1, Washington D.C., mimeo.

Valenzuela de Pisano, I. (1996), Agricultura y Bosque en Guatemala. Estudio de caso en Petén y Sierra de las Minas, Universidad Rafael Landívar et al., Guatemala.

Villar Anléu, L. (1998a), La flora silvestre de Guatemala, Colección Manuales núm. 6, Editorial Universitaria, Universidad de San Carlos, Guatemala.

Villar Anléu, L. (1998b), La fauna silvestre de Guatemala, Colección Manuales núm. 7, Editorial Universitaria, Universidad de San Carlos, Guatemala.

Vosti, S. y T. Reardon (1997), "Poverty-Environment Links in Rural Areas of Developing Countries", en Vosti, S. y T. Reardon (eds.): Sustainability, Growth, and Poverty Alleviation. A Policy and Agroecological Perspective, John Hopkins University Press, Baltimore, pp. 47-65.

Wells, M., K. Brandon y L. Hannah (1992), People and Parks. Linking Protected Area Management with Local Communities, Banco Mundial et al., Washington, D.C.

Wells, M., S. Guggenheim, A. Khan et al. (1999), Investing in Biodiversity: A Review of Indonesia's Integrated Conservation and Development Projects, Banco Mundial, Washington, D.C. 
Wilkie, J. W. (ed.) (1993), Statistical Abstract of Latin America, Los Angeles, UCLA Latin American Center Publications, núm. 30, partes 1 y 2 .

WRI (World Resources Institute) (1996), World resources 199697. A guide to the global environment, Oxford University Press, New York.

Enviado: 3 de febrero de 2003 Reenviado: 10 de abril de 2003 Aceptado: 18 de junio de 2003 
